\title{
Particulate versus non-particulate steroids for lumbar transforaminal or interlaminar epidural steroid injections: an update
}

\author{
Tobias J. Dietrich • Reto Sutter • Johannes M. Froehlich • \\ Christian W. A. Pfirrmann
}

\begin{abstract}
Received: 2 September 2014 /Revised: 24 October 2014 / Accepted: 28 October 2014 / Published online: 14 November 2014
\end{abstract}
(C) ISS 2014

\section{Introduction}

Lumbar transforaminal and interlaminar epidural steroid injections are widely used to treat patients with radicular pain and radiculopathy [1]. These lumbar spine procedures are reported to be clinically effective for short-term and longterm pain relief by some studies [2-5]. However, a relative lack of randomized and controlled clinical trials and as well as variability of the employed study methods were noted [6]. Thus, some controversy concerning the effectiveness of lumbar transforaminal and interlaminar epidural steroid injections still remains [6, 7]. Moreover, it should be emphasized that these indications and use are often performed on an off-label basis. The question arises whether just formal approval is lacking or whether extensive medical literature provides sufficient evidence-based efficacy and safety data for this offlabel use.

An image-guided therapeutic injection of both the cervical and lumbar spine is a minimally invasive technique, however rare serious adverse events such as brainstem, cerebellum and cervical, thoracic and lumbar spinal cord infarction have occurred [8-26]. The present article discusses serious complications associated with epidural steroid injections after injections at the level of the lumbosacral spine.

T. J. Dietrich $(\bowtie) \cdot$ R. Sutter $\cdot$ C. W. A. Pfirmann

Radiology, Orthopedic University Hospital Balgrist, Faculty of

Medicine, University of Zurich, Forchstrasse 340, 8008 Zurich, Switzerland

e-mail: tobiasjdietrich@gmail.com

J. M. Froehlich

ETH Zurich, Department of Chemistry and Applied Biosciences, Institute of Pharmaceutical Sciences, Vladimir-Prelog-Weg 1-5/10, 8093 Zurich, Switzerland
FDA safety announcement and off-label use

In 2011 the U.S. Food and Drug Administration (FDA) announced a safety labeling change for triamcinolone acetonide, warning that triamcinolone acetonide "is not for epidural use and to add information to the warning section that epidural use is not recommended" [27]. The product labeling of triamcinolone acetonide specifies therapeutic indications exclusively for intramuscular or intra-articular administration [28, 29]. Intravenous, intrathecal, epidural, and intraocular injections are listed as contraindication [28, 29]. In addition, the FDA released 2014 a safety announcement and stated "The FDA is warning that injection of corticosteroids into the epidural space of the spine may result in rare but serious adverse events, including loss of vision, stroke, paralysis, and death" [7]. The FDA informed patients and health care professionals: "The effectiveness and safety of epidural administration of corticosteroids have not been established, and FDA has not approved corticosteroids for this use" [7]. It was declared that the FDA will release an expert panel's recommendation addressing epidural steroid administration [7]. Thus, according to the FDA, epidural steroid injections in general have to be classified as off-label use, potentially hazardous and triamcinolone acetonide is even contraindicated for epidural injection.

\section{Criticism on the FDA safety announcement}

Manchikanti et al. [30] criticized the FDA warning in their review article. The authors stated that "multiple warnings and the initiation of alternate techniques to classic and traditional teachings, avoidance of particulate steroids, utilization of a blunt needle, and a multitude of other precautions have not been discussed or mentioned" by the FDA. In addition, the FDA neither differentiated between techniques such as transforaminal, interlaminar, and caudal epidural injections 
nor between the cervical, thoracic, and lumbar level. Manchikanti et al. [30] concluded: "The FDA should modify its statement, replacing it with an evidence-based warning emphasizing the off-label use of epidural steroids that can cause rare but serious neurologic problems following cervical and thoracic transforaminal epidural injections and also an increased risk with lumbar transforaminal epidural injections when performed without appropriate precautions" [30].

\section{What causes spinal cord damage?}

It was hypothesized that spinal cord infarction due to embolization of particulate steroids, needle-induced vasospasm, compression from an epidural hematoma or abscess and mechanical disruption of radiculomedullary arteries are possible mechanisms of spinal cord injury in patients undergoing cervical and lumbar epidural infiltrations [13-15, 31]. Noteworthy, benzyl alcohol, which is added as a preservative in many commercially available preparations, has been reported to be neurotoxic. Upon intrathecal injection, benzyl alcohol has been linked with paraplegia, neural degeneration, and demyelination [32-35]. However, it has to be stated that the pathophysiology of spinal cord injury remains unclear in most of these cases, in particular in patients after lumbosacral spine injections.

\section{Blood supply of the thoracolumbar spinal cord}

Radicular arteries are branches of the intercostal and lumbar arteries that are located within each neural foramen throughout the whole spine and mainly provide blood supply to the nerve root. A small number of radicular arteries with larger diameters also provide blood supply to the spinal cord, thus they are called radiculomedullary arteries. The largest anterior radiculomedullary artery, also known as the Adamkiewicz artery, supplies the lower two-thirds of the spinal cord via the anterior spinal artery [36-39]. The Adamkiewicz artery demonstrates a considerable anatomic variance. The Adamkiewicz artery originates in two-thirds of individuals from the left side and in one-third of individuals from the right as a branch of the intercostal and lumbar arteries, which arise from the aorta. A typical level of origin of the Adamkiewicz artery is at the intervertebral foramina between T6 and L2 [39, 40]. However, a lower level of feeding vessels to the anterior spinal artery was described between L3 and S2 [36, 37, 39, 41]. The inconstant Desproges-Gotteron artery arises from the internal iliac artery and was described as an anatomic variant of the L5 and S1 radicular artery, supplying both the nerve root and the spinal cord [42, 43].

Radicular arteries and radiculomedullary arteries, including the Adamkiewicz artery of the thoracolumbar spine, are most frequently found in the superoanterior part of the neural foramen (Fig. 1) and rarely in the inferior part of the neural foramen [38, 39]. Atluri et al. [44] reported that spinal cord infarction after transforaminal epidural steroid injection was related with a needle tip position within the superior and mid zone of the neural foramen, whereas needle tip position within the inferior part of the neural foramen was not observed in the cases with paralysis described in the literature.

Thus, it was suggested that inadvertent arterial needle puncture during transforaminal epidural steroid injection might be higher by the so-called safe triangle approach technique in the superoanterior part of the foramen (Fig. 2) compared to a needle tip location within the inferior aspect of the neural foramen (Fig. 3) [37-39, 44-46].

Two randomized, controlled, and prospective studies on lumbar transforaminal epidural steroid injections compared supra- and infraneural injection approaches in patients with radicular pain [47, 48]. Both studies demonstrated improvement of patients' outcome parameters with equivalent pain relief for the supra- and infraneural approach at 2-week and 12-week follow-up or 4- and 8-week follow-up, retrospectively $[47,48]$. However, neural contact of the needle was reported in $18 \%(9 / 50)$ of patients with the supraneural approach and none of 50 patients experienced neural contact with the infraneural approach [47]. Thus, an infraneural needle approach seems to be appropriate instead of a 'classic' supraneural or safe triangle approach, retrospectively, for lumbar transforaminal epidural steroid injections.

\section{Steroid preparations}

The allocation of particulate versus non-particulate steroid preparations is based on the solubility of the synthetic

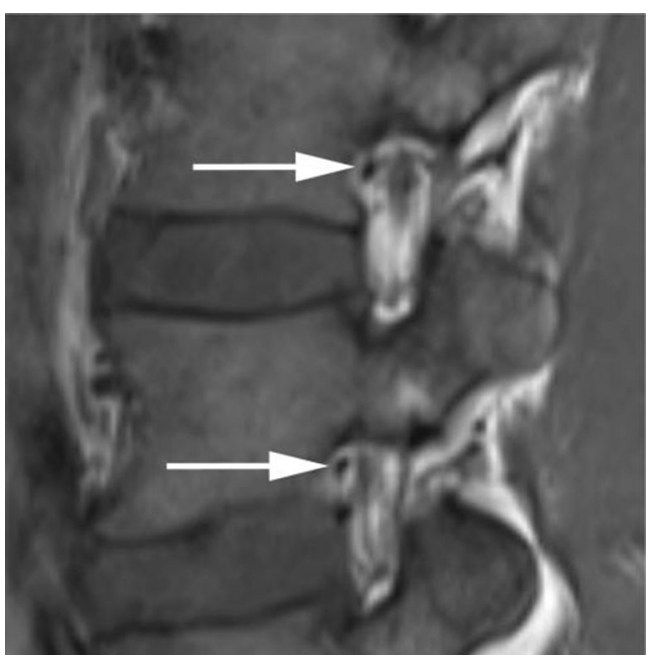

Fig. 1 Sagittal T1-weighted MR image demonstrates radicular arteries in the superoanterior part of the neural foramen L3-4 and L4-5 on the left side in a 38-year-old male patient 


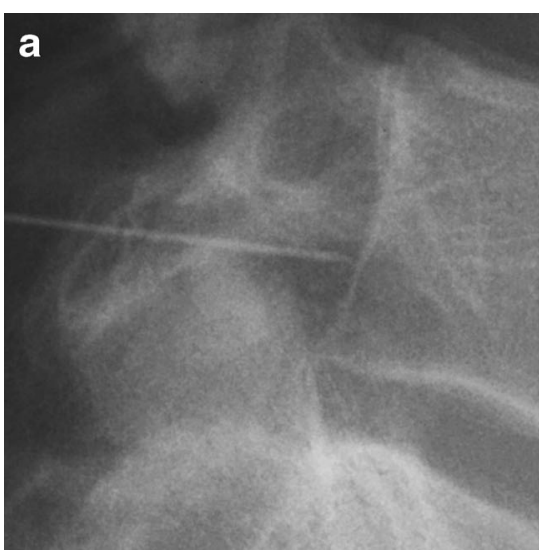

Fig. 2 A 24-year-old male patient underwent fluoroscopy-guided transforaminal epidural steroid injection by a supraneural approach to the L5 nerve root on the right side. Lateral (a) and anteroposterior oblique (b) radiograph demonstrates the injection needle tip in the superoanterior part of the neural foramen. b Iodinated contrast agent spreads along the

corticosteroids within water and secondarily on their aggregation characteristics. Particulate corticosteroids are usually poorly soluble in water, thus they might precipitate out of a solution and crystallize within a hydrophilic environment. Particulate steroids are available as lyophilisates provided in powder form needing reconstitution or as injectable suspensions, which contain solid particles sometimes in a mixture with a soluble corticosteroid. Most of the particles cover a size range between 0.5 and $100 \mu \mathrm{m}$, however sporadic particles even exceed a size of more than $1,000 \mu \mathrm{m}$ [49]. The following corticosteroids belong to the group of particulate steroids: triamcinolone acetonide, methylprednisolone acetate, betamethasone acetate, and prednisolone acetate. In contrast, dexamethasone sodium phosphate is considered as freely

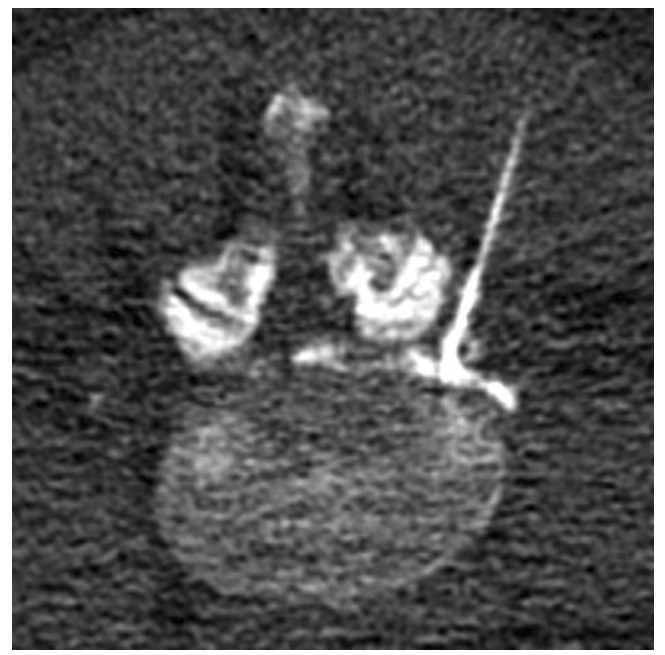

Fig. 3 A 57-year-old female patient underwent CT-guided transforaminal epidural steroid injection by an infraneural approach to the L4 nerve root on the right side. The transverse $\mathrm{CT}$ image demonstrates the injection needle tip in the inferoposterior part of the neural foramen. Iodinated contrast agent spreads along the nerve root into the epidural space. The image noise is related to a low-dose protocol $(120 \mathrm{kVp}$, $8 \mathrm{mAs}$ ) and the obesity of the patient

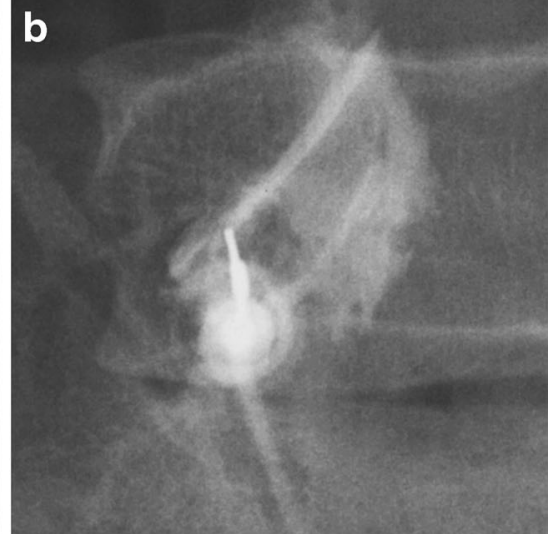

nerve root into the epidural space. It was reported that this 'classic' transforaminal epidural steroid injection approach by the so-called safe triangle technique is associated with a higher risk of spinal cord injury [44]

water-soluble. Thus, dexamethasone preparations are allocated to the non-particulate steroid group, despite the fact that some sporadic and small particles might be seen under the microscope with a typical particle size of $0.5 \mu \mathrm{m}[31,49,50]$. Betamethasone preparations are outliers of this binary classification: betamethasone preparations are commonly a mixture of betamethasone acetate (insoluble needing esterase activation) and betamethasone sodium phosphate (in solution) and provide characteristics of both groups, the particulate and nonparticulate steroids $[31,49,50]$. Moreover, there are also substantial differences in the propensity of different corticosteroid particles to aggregate into larger particles depending on the chemical ingredient (esters with larger particulate size), on the varying concentrations, on the drug vehicle, or on the drug mixtures with local anesthetics and/or contrast media prepared in-situ for pain treatment [50]. As a rule of thumb, one needs to be aware that incompatibilities with secondary precipitation might typically occur when mixing a larger anion (negative charge) with a larger cation (positive charge).

\section{Association between particle sizes and serious complications}

It was hypothesized that steroids with larger particle sizes might have a higher risk for occlusion of small vessels of the arterial tree as a potential consequence after incidental radiculomedullary artery steroid injection. Subsequently, infarction of the brainstem, cerebellum, and the spinal cord may occur. One might speculate that steroid particles smaller than the diameter of an erythrocyte are safer [31].

Dexamethasone sodium phosphate, triamcinolone acetonide, betamethasone sodium phosphate, betamethasone acetate, and methylprednisolone acetate injectable preparations were analyzed in vitro by light microscopy at 
magnification $(\times 400$ and $\times 1000)$ [31]. Derby et al. [31] have shown that the largest triamcinolone acetonide particles (range, $0.5-100 \mu \mathrm{m}$ ) were more than 12 times greater than median-sized erythrocytes (approximately $7.5 \mu \mathrm{m}$ ) [31]. Triamcinolone and betamethasone particles were densely packed with an extensive particle aggregation, e.g., some betamethasone aggregations were larger than $100 \mu \mathrm{m}$. Methylprednisolone alone and in a mixture with local anesthetics (lidocaine $\mathrm{HCl} 1 \%$ ) and iodinated contrast media (iohexol injection $240 \mathrm{mgI} / \mathrm{ml}$ ) revealed that particles and their aggregations were smaller than erythrocytes. However, methylprednisolone particles were densely packed, implicating the potential to form emboli with subsequent occlusion of small arterioles. Dexamethasone sodium phosphate particles were approximately ten times smaller than red blood cells without the tendency of particle aggregation. A mixture of dexamethasone, local anesthetic, and iodinated contrast media revealed the same properties [31] and did not precipitate.

Another research group evaluated methylprednisolone acetate, triamcinolone acetonide, and dexamethasone sodium phosphate [51] particle sizes of nondilute injectables and after mixing with local anesthetic and human plasma. Again, corticosteroids did not change their properties in a mixture with local anesthetics [51]. Additional plasma revealed a small but significant reduction in the size of aggregates eventually related to coating effects of albumin and repulsion effects; however, the size of the insoluble corticosteroid particles was unchanged [51]. The steroid aggregates maintained their integrity in a model replicating the flow of crystals within spinal cord arterioles, consequently the steroid aggregates might act as potential embolization agents [51]. Thus, the authors concluded that soluble dexamethasone is less likely to cause arterial or capillary occlusion after incidental intraarterial injection [31, 51].

\section{Injectable steroids in an animal model}

Particulate steroids (methylprednisolone acetate) and nonparticulate steroids (dexamethasone sodium phosphate, prednisolone sodium succinate) were injected into the vertebral artery of 11 swine while the animals were under general anesthesia [52]. All animals receiving intra-arterial particulate steroids failed to regain consciousness after the injection, required mechanical ventilation to replace spontaneous breathing, and MRI revealed cervical cord and brain stem edema. In addition, histopathology showed ischemic brain and spinal cord injury after the intra-arterial injection of particulate steroids consistent with a cerebrovascular insult. In contrast, animals that underwent intra-arterial injection of non-particulate steroids while under general anesthesia demonstrated full clinical recovery after the termination of general anesthesia and did not show any evidence of neurologic injury on MR images or histopathology [52]. This investigation in an animal model proposes that inadvertent intra-arterial injections of particulate steroids are associated with neurologic complications whereas intra-arterial non-particulate injections are safer compared to particulate steroids [52].

\section{Paraplegia in lumbosacral epidural injections}

Death as well as infarction of cerebellum, brain stem, thalamus, cervical, and lumbar spinal cord have been reported after cervical and lumbar epidural injections, either by an interlaminar or transforaminal approach [8-26]. In the literature, all 17 thoracolumbar spinal cord infarctions related to lumbosacral epidural steroid injection were associated with particulate steroids whereas no case of paraplegia was described after image-guided epidural injection of dexamethasone [16-26]. It has to be noted that the damage to the spinal cord commonly occurred several segments cranial to the lumbosacral injection site: for instance, in the case reported by Chang Chien et al., a transforaminal epidural steroid injection was performed at the level L5-S1, but subsequently spinal cord infarction on MRI was diagnosed between the T6 and the T9-T10 level [25]. All except for one [24] thoracolumbosacral spinal injection associated with ischemic complications described above were carried out as image-guided interventions, either by fluoroscopy or CT, and the use of complementary contrast agent application before steroid injection was common. Spinal cord injury occurred in 11 patients after fluoroscopy-guided [16, $18,20-23,25,26]$ and in four patients after CT-guided lumbar epidural steroid injections [16, 19, 21]. Even the combination of negative blood and cerebrospinal fluid aspiration with digital subtraction angiography (in order to exclude intravascular or intrathecal contrast medium spread) seems not to reliably exclude spinal cord infarction associated with lumbar transforaminal epidural steroid injection at the level of L5-S1 [25]. We assume that more lumbar epidural steroid injections are performed as fluoroscopy-guided injections compared to CT-guided injections. Thus there is no evidence to assess one image-guiding technique as safer or more dangerous compared to the other image-guiding technique for lumbar epidural steroid injections.

Fourteen thoracolumbar spinal cord infarctions occurred after transforaminal lumbar epidural steroid injections. Seven of these 14 transforaminal lumbar epidural steroid injection patients underwent previous lumbar spinal surgery. Three patients suffered from spinal cord injury after interlaminar lumbar epidural steroid injections and all of these patients underwent previous laminectomy below the segments of the injection $[22,24,26]$. There are no case reports or studies available of spinal cord injury associated with interlaminar lumbar epidural steroid injections in patients without previous lumbosacral spine surgery. Wybier et al. [22] stated that a 
remarkable high percentage of patients with spinal cord injury after lumbar epidural steroid injections underwent previous lumbar spine surgery. Thus, the authors speculated that the presence of epidural scar tissue increases the risk of spinal cord injury after lumbar epidural steroid injections [22].

The following corticosteroids were associated with ischemic stroke of the spinal cord or the posterior cerebral circulation after epidural spinal injections: triamcinolone acetonide, betamethasone, methylprednisolone acetate, and prednisolone acetate [8-26]. No patient in the literature suffered from an ischemic event after cervical or lumbosacral epidural imageguided injection of dexamethasone [8-26].

\section{Efficacy of various steroids in lumbar epidural injections}

It was hypothesized that particulate steroid preparations may provide a local depot effect with constant release of the active drug from the administration site over a longer time period compared to non-particulate steroids [53].

The duration of action of intramuscular administered triamcinolone acetonide representing a particulate glucocorticoid was studied in an investigation with dogs and compared to intravenous administered triamcinolone acetonide dihydrogen phosphate (TAA-DHP), which represents the non-particulate steroid [53]. It was shown that a single dose of the particulate glucocorticoid (triamcinolone acetonide) after intramuscular administration revealed a much longer duration of action of up to 4 weeks on the hypothalamicpituitary-adrenal axis activity in dogs than a similar intravenous dose of its analogue, the non-particulate steroid (TAADHP) [53]. The duration of action of the intravenous nonparticulate steroid (TAA-DHP) was only up to $72 \mathrm{~h}$ [53], although the potentially longer duration of action of intramuscular administered TAA-DHP was not evaluated in this study.

One may speculate that relief of symptoms might be lasting longer in patients after epidural injection of particulate steroids compared to non-particulate steroids. One study randomized a total of 106 lumbar transforaminal epidural steroid injection patients and compared the effectiveness of dexamethasone and triamcinolone acetate [54]. At 1-month follow-up the authors found a statistically significant lower visual analog pain score (VAS) in triamcinolone patients (reduction from VAS 8.3 to 2.4 ) compared to dexamethasone patients (reduction from 7.4 to 4.1). However, the two groups did not differ significantly on the McGill Pain Questionnaire, or the Oswestry Disability Index [54].

Three further studies compared the efficacy of a nonparticulate steroid (dexamethasone in all studies) and particulate steroids (methylprednisolone acetate, triamcinolone, or betamethasone) in lumbar transforaminal or interlaminar epidural steroid injections in patients with radicular pain or radiculopathy [55-57]. One of these three studies included retrospectively 3,645 transforaminal epidural steroid injections performed in 2,634 patients [56]. A second study on 78 consecutive patients was a prospective, randomized, and double-blind study [57]. The three studies mentioned above revealed no evidence that dexamethasone as a non-particulate steroid is less effective than particulate steroids in lumbar epidural injections [55-57]. It should be emphasized that these results were yielded despite non-equivalence of antiinflammatory effect based on corticosteroid potency despite dexamethasone in disadvantage. Kim and Brown [55] found a statistically nonsignificant trend towards less pain relief and shorter duration of action for dexamethasone in their retrospective study on a small cohort of 30 patients in each study group. In addition, Kennedy et al. [57] reported slightly more injections for each patient in the dexamethasone group with an average number of 1.6 injections compared to the triamcinolone group with an average number of 1.4 injections to achieve the same outcomes. However, $n=7(17 \%)$ patients of the dexamethasone group needed three injections compared to only $n=1$ ( $3 \%)$ patient of the triamcinolone group, which was the only statistically significant finding in the Kennedy et al. study [57]. Patient outcome after cervical transforaminal epidural steroid injection revealed similar findings compared to lumbar injections: particulate steroids did not demonstrate a superior effectiveness compared to non-particulate steroids $[58,59]$. Finally, it might be noted, that a prospective, randomized, double-blind, dose-response study on lumbar transforaminal epidural injections did not find a statistical difference in efficacy for dexamethasone dosage of $4 \mathrm{mg}$ compared with 8 or $12 \mathrm{mg}$ over a 12-week period [60]. The authors stated that the optimal dose of epidural dexamethasone might be even lower than $4 \mathrm{mg}[60]$.

\section{Conclusions}

In summary, all spinal cord infarctions related to lumbosacral epidural steroid injection in the literature were associated with particulate steroids and lumbar needle tip positions within the superior and mid zone of the neural foramen. In contrast, no case of paraplegia was described after image-guided epidural injection of soluble dexamethasone or a needle tip position within the inferior part of the neural foramen. Nevertheless, the pathophysiology of paraplegia associated with spinal cord infarction remains unclear, thus some experts still prefer particulate steroids in lumbar and thoracic transforaminal epidural steroid injections $[61,62]$. In this article, we present an update on the current literature regarding the use of corticosteroids for transforaminal epidural and interlaminar epidural injections. Despite the high evidence advocating the use of soluble corticosteroids due to paucity of well-conducted prospective studies, we are reluctant to provide general recommendations to health professionals. Corticosteroids present a 
marked variability in their formulations, secondarily mixtures might lead to aggregations or even drug incompatibilities and finally one cannot exclude vasoactive effects. Considering these pros and cons, at Balgrist University Hospital we have decided to administer $4 \mathrm{mg}$ of dexamethasone as a rapidly soluble steroid for lumbar transforaminal epidural and interlaminar epidural steroid pain injection placing the needle tip location in the inferior zone of the neural foramen according to evidence of the recent literature. Generally, particulate steroids are no longer employed at our institution for either transforaminal epidural or interlaminar epidural steroid injections. The administering radiologist should be aware of the still-limited efficacy data and potential adverse effects accompanying lumbar transforaminal or interlaminar epidural pain treatment with corticosteroids.

Conflict of interest Johannes M. Froehlich declares that he works as a consultant for Guerbet contrast media company.

Tobias J. Dietrich, Reto Sutter, and Christian W. A. Pfirrmann declare that they have no conflicts of interest. These authors had complete control of data and information.

\section{References}

1. Manchikanti L, Pampati V, Falco FJ, Hirsch JA. Assessment of the growth of epidural injections in the Medicare population from 2000 to 2011. Pain Physician. 2013;16:E349-64.

2. Manchikanti L, Buenaventura RM, Manchikanti KN, et al. Effectiveness of therapeutic lumbar transforaminal epidural steroid injections in managing lumbar spinal pain. Pain Physician. 2012;15: E199-245.

3. Kaufmann TJ, Geske JR, Murthy NS, et al. Clinical effectiveness of single lumbar transforaminal epidural steroid injections. Pain Med. 2013;14:1126-33.

4. Benyamin RM, Manchikanti L, Parr AT, et al. The effectiveness of lumbar interlaminar epidural injections in managing chronic low back and lower extremity pain. Pain Physician. 2012;15:E363-404.

5. MacVicar J, King W, Landers MH, Bogduk N. The effectiveness of lumbar transforaminal injection of steroids: a comprehensive review with systematic analysis of the published data. Pain Med. 2013;14: $14-28$.

6. Peterson C, Hodler J. Evidence-based radiology (part 1): Is there sufficient research to support the use of therapeutic injections for the spine and sacroiliac joints? Skeletal Radiol. 2010;39:5-9.

7. FDA Drug Safety Communication: FDA requires label changes to warn of rare but serious neurologic problems after epidural corticosteroid injections for pain. Available at: http://www.fda.gov/ downloads/Drugs/DrugSafety/UCM394286.pdf Accessed 2014 Aug 31 .

8. Hodler J, Boos N, Schubert M. Must we discontinue selective cervical nerve root blocks? Report of two cases and review of the literature. Eur Spine J. 2013;22 Suppl 3:S466-70.

9. Ludwig MA, Burns SP. Spinal cord infarction following cervical transforaminal epidural injection: a case report. Spine. 2005;30: E266-8.

10. Bose B. Quadriparesis following cervical epidural steroid injections: case report and review of the literature. Spine J. 2005;5:558-63.
11. Meyer HJ, Monticelli F, Kiesslich J. Fatal embolism of the anterior spinal artery after local cervical analgetic infiltration. Forensic Sci Int. 2005;149:115-9.

12. Popescu A, Lai D, Lu A, Gardner K. Stroke following epidural injections-case report and review of literature. J Neuroimaging. 2013;23:118-21.

13. Ziai WC, Ardelt AA, Llinas RH. Brainstem stroke following uncomplicated cervical epidural steroid injection. Arch Neurol. 2006;63: 1643-6.

14. Rozin L, Rozin R, Koehler SA, et al. Death during transforaminal epidural steroid nerve root block (C7) due to perforation of the left vertebral artery. Am J Forensic Med Pathol. 2003;24:351-5.

15. Wallace MA, Fukui MB, Williams RL, Ku A, Baghai P. Complications of cervical selective nerve root blocks performed with fluoroscopic guidance. AJR. 2007;188:1218-21.

16. Houten JK, Errico TJ. Paraplegia after lumbosacral nerve root block: report of three cases. Spine J. 2002;2:70-5.

17. Huntoon MA, Martin DP. Paralysis after transforaminal epidural injection and previous spinal surgery. Reg Anesth Pain Med. 2004;29:494-5.

18. Glaser SE, Falco F. Paraplegia following a thoracolumbar transforaminal epidural steroid injection. Pain Physician. 2005;8: 309-14.

19. Somayaji HS, Saifuddin A, Casey AT, Briggs TW. Spinal cord infarction following therapeutic computed tomography-guided left L2 nerve root injection. Spine. 2005;30:E106-8.

20. Quintero N, Laffont I, Bouhmidi L, et al. Transforaminal epidural steroid injection and paraplegia: case report and bibliographic review. Ann Readapt Med Phys. 2006;49:242-7.

21. Kennedy DJ, Dreyfuss P, Aprill CN, Bogduk N. Paraplegia following image-guided transforaminal lumbar spine epidural steroid injection: two case reports. Pain Med. 2009;10:1389-94.

22. Wybier M, Gaudart S, Petrover D, Houdart E, Laredo JD. Paraplegia complicating selective steroid injections of the lumbar spine. Report of five cases and review of the literature. Eur Radiol. 2010;20:181-9.

23. Lyders EM, Morris PP. A case of spinal cord infarction following lumbar transforaminal epidural steroid injection: MR imaging and angiographic findings. AJNR. 2009;30:1691-3.

24. Thefenne L, Dubecq C, Zing E, et al. A rare case of paraplegia complicating a lumbar epidural infiltration. Ann Phys Rehabil Med. 2010;53:575-83.

25. Chang Chien GC, Candido KD, Knezevic NN. Digital subtraction angiography does not reliably prevent paraplegia associated with lumbar transforaminal epidural steroid injection. Pain Physician. 2012;15:515-23.

26. Lenoir T, Deloin X, Dauzac C, Rillardon L. Guigui P [Paraplegia after interlaminar epidural steroid injection: a case report]. Rev Chir Orthop Reparatrice Appar Mot. 2008;94:697-701.

27. FDA - U.S. Food and Drug Administration. Shoulder. Kenalog-10 (triamcinolone acetonide) injection and Kenalog-40 (triamcinolone acetonide) injection. Available at: http://www.fda.gov/safety/ medwatch/safetyinformation/ucm262876.htm Accessed 2014 Aug 31.

28. Electronic Medicines Compendium. Kenalog Intra-articular / Intramuscular Injection. Summary of Product Characteristics (last updated on the eMC: 06/05/2014) Available at: http://www. medicines.org.uk/emc/medicine/11366/SPC/Kenalog+Intraarticular+++Intramuscular+Injection Accessed 2014 Aug 31.

29. DailyMed. Original Packager: KENALOG-40 (triamcinolone acetonide) injection, suspension Available at: http://dailymed.nlm. nih.gov/dailymed/lookup.cfm?setid=cf970688-cd77-b95c-4bd9c541590e9722 Accessed 2014 Aug 31.

30. Manchikanti L, Candido KD, Singh V, Gharibo CG, Boswell MV, Benyamin RM, et al. Epidural steroid warning controversy still dogging FDA. Pain Physician. 2014;17:E451-74. 
31. Derby R, Lee SH, Date ES, Lee JH, Lee CH. Size and aggregation of corticosteroids used for epidural injections. Pain Med. 2008;9:22734.

32. Craig DB, Habib GG. Flaccid paraparesis following obstetrical epidural anesthesia: possible role of benzyl alcohol. Anesth Analg. 1977;56:219-21.

33. Hahn AF, Feasby TE, Gilbert JJ. Paraparesis following intrathecal chemotherapy. Neurology. 1983;33:1032-8.

34. Feasby TE, Hahn AF, Gilbert JJ. Neurotoxicity of bacteriostatic water. N Engl J Med. 1983;308:966-7.

35. Hetherington NJ, Dooley MJ. Potential for patient harm from intrathecal administration of preserved solutions. Med J Aust. 2000;173: $141-3$.

36. Romanes GJ. The arterial blood supply of the human spinal cord. Paraplegia. 1965;2:199-207.

37. Biglioli P, Roberto M, Cannata A, et al. Upper and lower spinal cord blood supply: the continuity of the anterior spinal artery and the relevance of the lumbar arteries. J Thorac Cardiovasc Surg. 2004;127:1188-92.

38. Murthy NS, Maus TP, Behrns CL. Intraforaminal location of the great anterior radiculomedullary artery (artery of Adamkiewicz): a retrospective review. Pain Med. 2010;11:1756-64.

39. Kroszczynski AC, Kohan K, Kurowski M, Olson TR, Downie SA. Intraforaminal location of thoracolumbar anterior medullary arteries. Pain Med. 2013;14:808-12.

40. Boll DT, Bulow H, Blackham KA, Aschoff AJ, Schmitz BL. MDCT angiography of the spinal vasculature and the artery of Adamkiewicz. AJR. 2006;187:1054-60.

41. Lo D, Valleé JN, Spelle L, et al. Unusual origin of the artery of Adamkiewicz from the fourth lumbar artery. Neuroradiology. 2002;44:153-7.

42. Reis C, Rocha JA, Chamadoira C, Pereira P, Fonseca J. Foraminal L5-S1 disc herniation and conus medullaris syndrome: a vascular etiology? Acta Neurochir. 2007;149:533-5.

43. Masson C, Bardin T. Desproges-Gotteron artery, spinal cord infarction, and transforaminal glucocorticoid injections. Joint Bone Spine. 2009; 76:120-1.

44. Atluri S, Glaser SE, Shah RV, Sudarshan G. Needle position analysis in cases of paralysis from transforaminal epidurals: consider alternative approaches to traditional technique. Pain Physician. 2013;16: 321-34.

45. Zhu J, Falco FJ, Formoso F, Onyewu O, Irwin FL. Alternative approach for lumbar transforaminal epidural steroid injections. Pain Physician. 2011;14:331-41.

46. Glaser SE, Shah RV. Root cause analysis of paraplegia following transforaminal epidural steroid injections: the 'unsafe' triangle. Pain Physician. 2010;13:237-44.

47. Park KD, Lee J, Jee H, Park Y. Kambin triangle versus the supraneural approach for the treatment of lumbar radicular pain. Am J Phys Med Rehabil. 2012;91:1039-50.

48. Park $\mathrm{CH}$, Lee SH, Park HS. Lumbar retrodiscal versus postganglionic transforaminal epidural steroid injection for the treatment of lumbar intervertebral disc herniations. Pain Physician. 2011;14: 353-60.

49. Benzon HT, Chew TL, McCarthy RJ, Benzon HA, Walega DR. Comparison of the particle sizes of different steroids and the effect of dilution: a review of the relative neurotoxicities of the steroids. Anesthesiology. 2007;106:331-8.

50. MacMahon PJ, Eustace SJ, Kavanagh EC. Injectable corticosteroid and local anesthetic preparations: a review for radiologists. Radiology. 2009;252:647-61.

51. MacMahon PJ, Shelly MJ, Scholz D, Eustace SJ, Kavanagh EC. Injectable corticosteroid preparations: an embolic risk assessment by static and dynamic microscopic analysis. AJNR. 2011;32:1830-5.

52. Okubadejo GO, Talcott MR, Schmidt RE, et al. Perils of intravascular methylprednisolone injection into the vertebral artery. An animal study. J Bone Joint Surg Am. 2008;90:1932-8.

53. Abraham G, Demiraj F, Ungemach FR. Comparison of the hypothalamic-pituitary-adrenal axis susceptibility upon single-dose i.m. depot versus long-acting i.v. triamcinolone acetonide therapy: a direct pharmacokinetic correlation. J Endocrinol. 2006;191:491-6.

54. Park CH, Lee SH, Kim BI. Comparison of the effectiveness of lumbar transforaminal epidural injection with particulate and nonparticulate corticosteroids in lumbar radiating pain. Pain Med. 2010;11:1654-8.

55. Kim D, Brown J. Efficacy and safety of lumbar epidural dexamethasone versus methylprednisolone in the treatment of lumbar radiculopathy: a comparison of soluble versus particulate steroids. Clin J Pain. 2011;27:518-22.

56. El-Yahchouchi C, Geske JR, Carter RE, et al. The noninferiority of the nonparticulate steroid dexamethasone vs the particulate steroids betamethasone and triamcinolone in lumbar transforaminal epidural steroid injections. Pain Med. 2013;14:1650-7.

57. Kennedy DJ, Plastaras C, Casey E, et al. Comparative effectiveness of lumbar transforaminal epidural steroid injections with particulate versus nonparticulate corticosteroids for lumbar radicular pain due to intervertebral disc herniation: a prospective, randomized, doubleblind trial. Pain Med. 2014;15:548-55.

58. Lee JW, Park KW, Chung SK, et al. Cervical transforaminal epidural steroid injection for the management of cervical radiculopathy: a comparative study of particulate versus non-particulate steroids. Skeletal Radiol. 2009;38:1077-82.

59. Shakir A, Ma V, Mehta B. Comparison of pain score reduction using triamcinolone vs. dexamethasone in cervical transforaminal epidural steroid injections. Am J Phys Med Rehabil. 2013;92:768-75.

60. Ahadian FM, McGreevy K, Schulteis G. Lumbar transforaminal epidural dexamethasone: a prospective, randomized, double-blind, dose-response trial. Reg Anesth Pain Med. 2011;36:572-8.

61. Shah RV. Paraplegia following thoracic and lumbar transforaminal epidural steroid injections: how relevant are particulate steroids? Pain Pract. 2014;14:297-300.

62. DePalma MJ, Stout A, Kennedy DJ. Corticosteroid choice for epidural injections. PM R. 2013;5:524-32. 